\title{
A Unified Real-time Automatic Congestion Identification Model Considering Weather and Roadway Visibility Conditions
}

\author{
Mohammed Elhenawy, Hesham Rakha and Hao Chen \\ Virginia Tech Transportation Institute, 3500 Transportation Research Plaza, Blacksburg, VA 24061, U.S.A.
}

Keywords: Transportation Planning and Traffic Operation, Real-time Automatic Congestion Identification, Mixture of Linear Regression, ITS.

\begin{abstract}
Real-time automatic congestion identification is one of the important routines of intelligent transportation systems (ITS). Previous efforts usually use traffic state measurements (speed, flow, occupancy) to develop congestion identification algorithms. However, the impacts of weather conditions to identify congestion have not been investigated in the existing studies. In this paper, we proposed an algorithm that uses the speed probe data and the corresponding weather and visibility to build a transferable model. This model can be used on any road stretch. Our algorithm assumes traffic states can be classified into three regimes: congestion, speed at capacity and free-flow. Moreover, the speed distribution follows a mixture of three components whose means are functions in weather and visibility. The mean of each component is defined using a linear regression using different weather conditions and visibility levels as predictors. We used three data sets from VA, CA and TX to estimate the model parameters. The fitted model is used to calculate the speed cut-off between congestion and speed at capacity which minimize either the Bayesian classification error or the false positive (congestion) rate. The test results demonstrate the proposed method produces promising congestion identification output by considering weather condition and visibility.
\end{abstract}

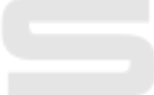

\section{1 -INTRODUCTION}

Traffic congestion has become one of the modern life problems in many metropolitan areas. This growing problem has environmental effects. During congestion time, cars cannot run efficiently so air pollution, carbon dioxide (CO2) emissions, and fuel use increase. In 2007, Americans lost $\$ 87.2$ billion in wasted fuel and lost productivity. This waste reached \$115 billion in 2009 (Ibrahim and Hall, 1994). Congestion increases travel time, for example back in 1993 driving under congested condition causes a delay of about six-tenths of a minute per kilometre of travel on expressways and 1.2 minutes delay per kilometre of travel in arterials(Arnott and Small, 1994). The congestion problem becomes worse as reported by Texas Transportation Institute where the number of Americans' wasted hours in traffic congestion becomes fivefold between 1982 and 2005 Moreover, congestion has its economic effect where studies show that congestion slow metropolitan growth, inhibits agglomeration economies, and shape economic geographies(Sweet, 2011). Traffic Congestion could result by an obstruction or lack of road capacity which is a kind of inefficient use of the roads. This problem can be relaxed by increasing the road-building budgets to build more infrastructures. But adding more road capacity is costly and budget is limited, and the construction itself takes a long time. With the continuous increase in traffic volumes, managing traffic, particularly at times of peak demand, is a good and inexpensive solution to congestion. Advanced traffic management systems (ATMS) use various applications of intelligent transportation systems (ITS) to manage traffic and reduce congestion problems. Recently, the advancement in communication and computers greatly improve ITS and make it more capable of identifying and reducing congestion. ITS is an effective solution to traffic problem where it improves the dynamic capacity of the road system without building extra expensive infrastructure (Jianming et al., 2012). Accurate and real-time traffic information is the foundation of ITS.

Congestion usually starts from a road bottleneck, then spills over the neighbour road segments. It takes time until this congestion disappears. Depending on the frequency of congestion occurrence, traffic congestion can be divided into two categories (Guiyan et al., 2010). The first is recurrent traffic 
congestion, and the second is accidental (nonrecurring) traffic congestion. Recurrent traffic congestion, which usually results from exceeding the road capacity, is easier to identify and predict. The accidental traffic congestion usually results from traffic incident or severe weather conditions. Traffic congestion is different at different locations, time periods, and different weather conditions.

The impact of weather on the freeway traffic operations is a big concern for roadway management agencies, however, there is little research done to link weather and congestion in a quantitative sense. Two groups at the University of Washington correlated weather and traffic phenomena using the Traffic Data Acquisition and Distribution (TDAD) data mine and the Doppler radar data mine (Dailey, 2006). Their basic idea is that, moving weather cells can be tracked and predicted using weather radar then they can find the correlation between the properties of the weather cell and observed traffic states. Nookala studied the traffic congestion caused by weather conditions and its effect on traffic volume and travel time (Nookala, 2006). He observed an increase in the traffic congestion at inclement weather conditions due to drop in the freeway capacity while the traffic demand does not drop significantly. Chung et al. used traffic data collected over a 2 year period from July first 2002 to June thirty 2004 at Tokyo Metropolitan Expressway (MEX) and showed a decrease in free flow speed and in capacity with increasing amount of rainfall(Chung et al., 2006). Brilon and Ponzlet used three years of historical data for 15 freeway sites in Germany to investigate impacts of several factors including weather on speed-flow relationships (Brilon and Ponzlet, 1996). They found that wet roadway conditions cause different speed reduction at highways with different lane number. Agarwal et al. highlighted that the results obtained from studies outside the United States can't applied within the United States due to the different roadway and driver characteristics. Moreover, the result obtained from rural freeway segments within the United States may be different from urban freeway(Agarwal et al., 2005). Ibrahim and Hall used limited historical data set and multiple regression analysis to study the impact of rain and snow on speed (Ibrahim and Hall, 1994). Their results showed that light rain and snow causes similar reductions in speeds $(3 \%-5 \%)$, while $14 \%-15 \%$ and $30 \%-40 \%$ reduction in speed are caused by heavy rain and heavy snow respectively. Rakha et al used weather data (precipitation and visibility) and loop detector data (speed, flow, and occupancy) obtained from Baltimore, Twin Cities, and Seattle in the USA to quantify the impact of inclement weather on traffic stream behavior and key traffic stream parameters, including free-flow speed, speed-at-capacity, capacity, and jam density. For more detailed discussion of the Rakha's result readers are referred to (Rakha et al., 2007).

During the last few years, many automatic congestion identification algorithms are proposed. ASBIA is an algorithm that uses speed measurements over short temporal and spatial intervals and segments, respectively to identify the status of a segment using t-test(Elhenawy et al., 2013). The outputs of the algorithm are the status of the roadway segment (free-flow or congested) and the confidence level of the test ( $p$-value). Another algorithm uses vehicle trajectories in intelligent vehicle infrastructure co-operation system (IVICS)(Jianming et al., 2012). Then the spatial-temporal trajectories are considered as an image to extract the propagation speed of congestion wave and construct congestion template. Finally correlation is evaluated between the template and the spatial-temporal velocity image to identify the congestion. Parallel SVM is used in (Sun et al., 2012) to identify traffic congestion. The authors propose Parallel SVM instead of SVM because the training computation cost of SVM is expensive and congestion identification is a real-time task.

Floating car data is used in (Xu et al., 2013) to find meaningful congestion patterns. The analysis of the floating car data is done using a method based on data cube and the spatial-temporal related relationship of the slow-speed road segment to identify the traffic congestion. The research team at the Center for Sustainable Mobility (CSM) at the Virginia Tech Transportation institute (VTTI) developed an algorithm to identify congested segments using a spatiotemporal speed matrix (Elhenawy and Rakha, 2013). The proposed algorithm fits two log-normal (or normal) distributions to the training dataset.

To the best of our knowledge, no research addresses the impacts of both visibility and weather conditions on congestion identification. In this paper, the impacts of weather conditions and visibility levels on the congestion identification algorithm are investigated by modelling the speed distribution as mixture of three log-normal components whose means are linear function of weather condition and visibility level. So that based on these factors the three log-normal components may get close or apart and the cut-off speed is changed. The proposed algorithm is built using three different data set from three different states (VA, TX and CA). The results of our proposed model are promising and reasonable where, for example, the cut-off speed increases as the visibility level increases. 
The remainder of this paper is organized as follows. First, a brief background of the method used in this work is given. After that, the proposed algorithm is introduced. The data sets used in the case study is described. Subsequently the result of the experimental work is explained and an illustrative example is given to show how to implement the proposed model. Finally, conclusions and recommendations for future work are presented.

\section{MIXTURE OF LINEAR REGRESSIONS}

Finite mixture models are powerful tools in analyzing a wide variety of random phenomena. They are used to model random phenomena in many fields including agriculture, biology, economics, medicine and genetics. A mixture of linear regressions is one of the mixture families studied carefully in the literature (De Veaux, 1989, Faria and Soromenho, 2009). It can be used to model the speed for different traffic regimes at different weather condition and visibility levels.

The mixture of linear regression can be written as:

$$
\text { Or as } p(y \mid X)=\sum_{j=1}^{m} \frac{\lambda_{j}}{\sigma_{j} \sqrt{2 \pi}} e^{-\frac{\left(y-x^{T} \beta_{j}\right)^{2}}{2 \sigma_{j}^{2}}}
$$

$$
\mathrm{y}_{\mathrm{i}}=\left\{\begin{array}{c}
\mathrm{x}_{\mathrm{i}}^{\mathrm{T}} \beta_{1}+\epsilon_{\mathrm{i} 1} \text { with probability } \lambda_{1} \\
\mathrm{x}_{\mathrm{i}}^{\mathrm{T}} \beta_{2}+\epsilon_{\mathrm{i} 2} \text { with probability } \lambda_{2} \\
\cdot \\
\cdot \\
\cdot \\
\mathrm{x}_{\mathrm{i}}^{\mathrm{T}} \beta_{\mathrm{m}}+\epsilon_{\mathrm{im}} \text { with probability } 1-\sum_{\mathrm{q}=1}^{\mathrm{m}-1} \lambda_{\mathrm{q}}
\end{array}\right.
$$

where $y_{\mathrm{i}}$ is a response corresponding to a predictors' vector $\mathrm{x}_{\mathrm{i}}^{\mathrm{T}}, \beta_{\mathrm{j}}$ is a vector of regression coefficients for the $\mathrm{j}^{\text {th }}$ mixture component, $\lambda_{\mathrm{j}}$ is a mixing probability of the $\mathrm{j}^{\text {th }}$ mixture component, $\epsilon_{\mathrm{ij}}$ are normal random errors, and $\mathrm{m}$ is the number of components in mixture model. Model parameters $\psi=\left\{\beta_{1}, \beta_{2}, \ldots, \beta_{\mathrm{m}}, \sigma_{1}^{2}, \sigma_{2}^{2}, \ldots, \sigma_{\mathrm{m}}^{2}, \lambda_{1}, \lambda_{2}, \ldots, \lambda_{\mathrm{m}}\right\}$ can be estimated by maximizing the log-likelihood of Equation (1); given a set of response predictor pairs $\left(\mathrm{y}_{1}, \mathrm{x}_{1}\right),\left(\mathrm{y}_{2}, \mathrm{x}_{2}\right), \ldots,\left(\mathrm{y}_{\mathrm{n}}, \mathrm{x}_{\mathrm{n}}\right)$, and using the Expectation-Maximization algorithm (EM).

\subsection{EM Algorithm}

The EM algorithm iteratively finds maximum likelihood estimates by alternating the E-step and M- step. Let $\psi^{(\mathrm{k})}$ be parameter estimates after the $\mathrm{k}^{\text {th }}$ iteration. On the E-step, the posterior probability of the $\mathrm{i}^{\text {th }}$ observation comes from component $\mathrm{j}$ and is computed as shown in Equation (3).

$$
\mathrm{w}_{\mathrm{ij}}^{(\mathrm{k}+1)}=\frac{\lambda_{\mathrm{j}}^{(\mathrm{k})} \phi_{\mathrm{j}}\left(\mathrm{y}_{\mathrm{i}} \mid \mathrm{x}_{\mathrm{i}}, \psi^{(\mathrm{k})}\right)}{\sum_{\mathrm{j}=1}^{\mathrm{m}} \lambda_{\mathrm{j}}^{(\mathrm{k})} \phi_{\mathrm{j}}\left(\mathrm{y}_{\mathrm{i}} \mid \mathrm{x}_{\mathrm{i}}, \psi^{(\mathrm{k})}\right)}
$$

where $\phi_{j}\left(y_{i} \mid x_{i}, \psi^{(k)}\right)$ is the probability density function of the $\mathrm{j}^{\text {th }}$ component

On the M-step, new parameter estimates $\psi^{(\mathrm{k}+1)}$ maximizing the log-likelihood function in Equation (1) are calculated, as shown in Equations (4-5).

$$
\begin{gathered}
\lambda_{j}^{(k+1)}=\frac{\sum_{i=1}^{n} w_{i j}^{(k+1)}}{n} \\
\widehat{\beta}_{j}^{(k+1)}=\left(X^{T} W_{j} X\right)^{-1} X^{T} W_{j} Y
\end{gathered}
$$

where $\mathrm{X}$ is an $\mathrm{nx}(\mathrm{p}+1)$ predictor matrix, $\mathrm{Y}$ is the corresponding $n \times 1$ response vector, and $W$ is an $n \times n$ diagonal matrix having $\mathrm{w}_{\mathrm{ij}}^{(\mathrm{k}+1)}$ along its diagonal

$$
\widehat{\sigma}_{j}^{2(k+1)}=\frac{\sum_{i=1}^{n} w_{i j}^{(k+1)}\left(y_{i}-x_{i}^{T} \widehat{\beta}_{j}^{(k+1)}\right)^{2}}{\sum_{i=1}^{n} w_{i j}^{(k+1)}}
$$

The E-step and M-step are alternated repeatedly until the incomplete log-likelihood change is arbitrarily small, as shown in Equation (7).

$$
\begin{array}{r}
\mid \prod_{\mathrm{i}=1}^{\mathrm{n}} \sum_{\mathrm{j}=1}^{\mathrm{m}} \lambda_{\mathrm{j}}^{(\mathrm{k}+1)} \phi_{\mathrm{j}}\left(\mathrm{y}_{\mathrm{i}} \mid \mathrm{x}_{\mathrm{i}}, \psi^{(\mathrm{k}+1)}\right)- \\
\prod_{\mathrm{i}=1}^{\mathrm{n}} \sum_{\mathrm{j}=1}^{\mathrm{m}} \lambda_{\mathrm{j}}^{(\mathrm{k})} \phi_{\mathrm{j}}\left(\mathrm{y}_{\mathrm{i}} \mid \mathrm{x}_{\mathrm{i}}, \psi^{(\mathrm{k})}\right) \mid<\xi
\end{array}
$$

where $\xi$ is a small number

\section{PROPOSED ALGORITHM}

As shown in the following fundamental diagrams, we divide the traffic states of a road segment into three traffic regimes where the speed of each regime can be modeled by a log-normal distribution. So that the overall speed distribution can be represented as a mixture of three log-normal components. First regime is the free flow which has the speed distribution with the highest mean. At free flow regime, the density lies below the capacity density. The second regime is the Congested flow which has the speed distribution with the lowest mean. The congested flow is characterized by the traffic that has density lies between the capacity density and the jam density. The third regime is the capacity flow which separates the free flow from the congested flow and its speed distribution has a mean that between the means of the other two regimes. As shown in several studies the flow fundamental diagram is affected by the weather conditions (Meead Saberi and Bertini, 
Smith et al., 2003, Rakha et al., 2007). So that we expect the mean of the speed distribution corresponding to each regime changes with weather and visibility. The proposed algorithm uses the mixture of three linear regression and real data sets to learn the means of the distribution as a function of weather and visibility and find the boundary between the three regimes. The proposed algorithm is shown below.
All segments with speeds greater than the threshold are classified as free-flow segments, and other segments are classified as congested segments. The output of the above algorithm is a spatiotemporal binary matrix with dimensions identical to the spatiotemporal speed matrix. A ' 1 ' in the binary matrix identifies a segment as congested, and a ' 0 ' represents free-flow conditions.

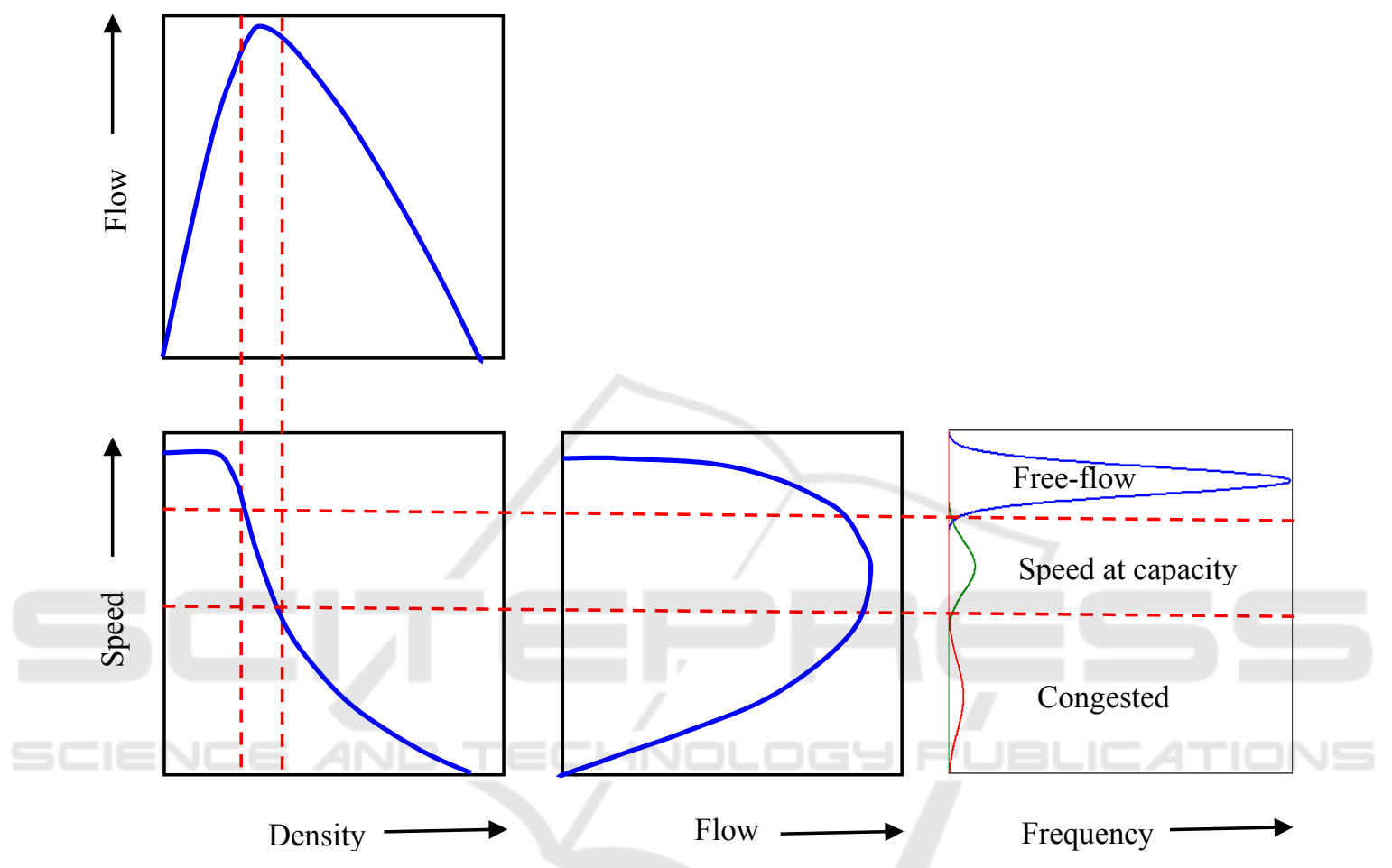

Figure 1: Illustration of Link between the Fundamental Diagrams and the Three Components Mixture.

Table 1: The proposed algorithm.

1. Use the EM algorithm described earlier to fit three component distributions to locally-collected data, as demonstrated in Equation (8).

$$
\begin{aligned}
& \left(\log (\mathrm{y}) \mid \lambda_{1}, \lambda_{2}, \beta_{1}, \beta_{2}, \beta_{3}, \sigma_{1}, \sigma_{2}, \sigma_{3}\right)=\lambda_{1} \frac{1}{\sqrt{2 \pi} \sigma_{1}} \mathrm{e}^{\frac{\left(\log (y)-\mathrm{x}^{\mathrm{T}} \beta_{1}\right)^{2}}{2 \sigma_{1}{ }^{2}}}+\lambda_{2} \frac{1}{\sqrt{2 \pi} \sigma_{2}} \mathrm{e}^{\frac{\left(\log (\mathrm{y})-\mathrm{x}^{\mathrm{T}} \beta_{2}\right)^{2}}{2 \sigma_{2}{ }^{2}}}+\left(1-\lambda_{2}-\right. \\
& \left.\lambda_{1}\right) \frac{1}{\sqrt{2 \pi} \sigma_{3}} \mathrm{e}^{\frac{\left(\log (\mathrm{y})-\mathrm{x}^{\mathrm{T}} \beta_{3}\right)^{2}}{2 \sigma_{3}{ }^{2}}}, \quad \text { (8) }
\end{aligned}
$$

Where vector $X$ is a vector of weather conditions and visibility predictors.

Here $\left(\mathrm{X}^{\mathrm{T}} \beta_{1}, \sigma_{1}\right),\left(\mathrm{X}^{\mathrm{T}} \beta_{2}, \sigma_{2}\right)$, and $\left(\mathrm{X}^{\mathrm{T}} \beta_{3}, \sigma_{3}\right)$ are the locations and spreads of the mixture components, and $\left(\lambda_{1}, \lambda_{2}\right)$ are the mixture parameters.

2. For unseen data use the weather condition, visibility level, and equations of the means $\left(\mathrm{X}^{\mathrm{T}} \beta_{1}, \mathrm{X}^{\mathrm{T}} \beta_{2}\right.$, and $\left.\mathrm{X}^{\mathrm{T}} \beta_{3}\right)$ to calculate locations (means) of three components.

3. Calculate the cut-off speed. We have two options to calculate the cut-off speed and we can use either of them.

3.1. Calculate 0.001 quintile of the speed at capacity (the middle distribution).

3.2. Calculate cut-off speed using the Bayesian approach; which finds the intersection point (between congestion and speed at capacity) that minimizes classification error(Elhenawy et al., 2015).

4. Use cut-off speed as a threshold to classify the state of each road segment. 


\section{EXPERIMENTAL WORK}

\subsection{Data Reduction}

In order to use collected traffic data in the proposed algorithm, data reduction was an important process for transferring raw measured data into required input data formats. In general, the spatiotemporal traffic state matrix is a fundamental attribute of input data. Reduction of INRIX probe data is one example, and a similar process can be applied to other types of measured data (e.g. loop detector). INRIX data are collected for each roadway segment and time interval. Each roadway segment represents a TMC station. Geographic TMC station information is also provided. The average speed for each TMC station can be used to derive a spatiotemporal traffic state matrix. However, raw INRIX data includes geographically inconsistent sections, irregular time intervals of data collection, and missing data. Considering these problems, the data reduction process is illustrated in Figure 2.

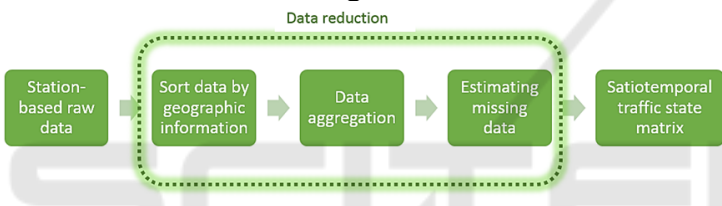

Figure 2: Data Reduction of INRIX Probe Data

Based on the geographic information of each TMC station, raw data are sorted along the roadway direction (e.g. towards eastbound or westbound). An examination should be adopted to check any overlapping or inconsistent stations along the direction. Afterwards, speed data should be aggregated by time intervals (e.g. 5 minutes), according to the algorithm's resolution requirement. In this way, raw data can be aggregated into a daily matrix format, along spatial and temporal intervals. It should be noted that missing data usually exist on the developed data matrix. Therefore, data imputation methods should be conducted, to estimate the missing data by neighbouring cell values. Consequently, the daily spatiotemporal traffic state matrix can be generated for congestion and bottleneck identification.

\subsection{Study Sites}

INRIX traffic data in three states (Virginia, Texas and California) were used to develop the proposed automatic congestion identification algorithm. Specifically, the study included 2011 2013 data along I-66 eastbound, 2012 data along US-75 northbound and 2012 data along I-15 southbound. The selected freeway corridor on I-66 is presented in Figure 3, which includes 36 freeway segments along 30.7 miles. Average speeds (or travel times) for each roadway segment are provided in the raw data, which were collected every minute. In order to reduce the stochastic noise and measurement error, raw speed data were aggregated by five-minute intervals. Therefore, the traffic speed matrix over spatial (upstream to downstream) and temporal (from 0:00 AM to 23:55 PM) domains could be obtained for each day. For the other two locations, daily speed matrices were obtained using the same procedure. Selected freeway corridors on US-75 and I-15 are presented in Figure 4 and Figure 5; including 81 segments across 38 miles, and 30 segments across 15.6 miles, respectively.

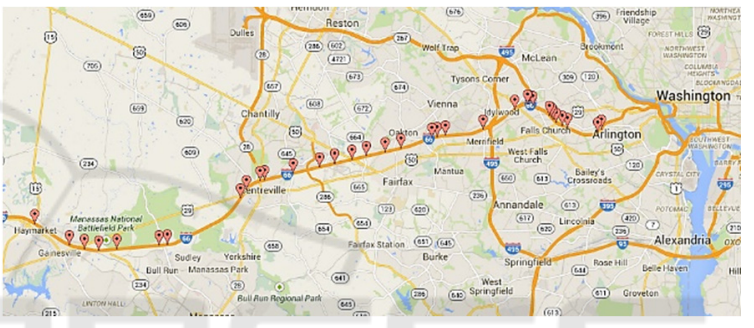

Figure 3: Layout of the Selected Freeway Stretch on I-66. (Source: Google Maps).

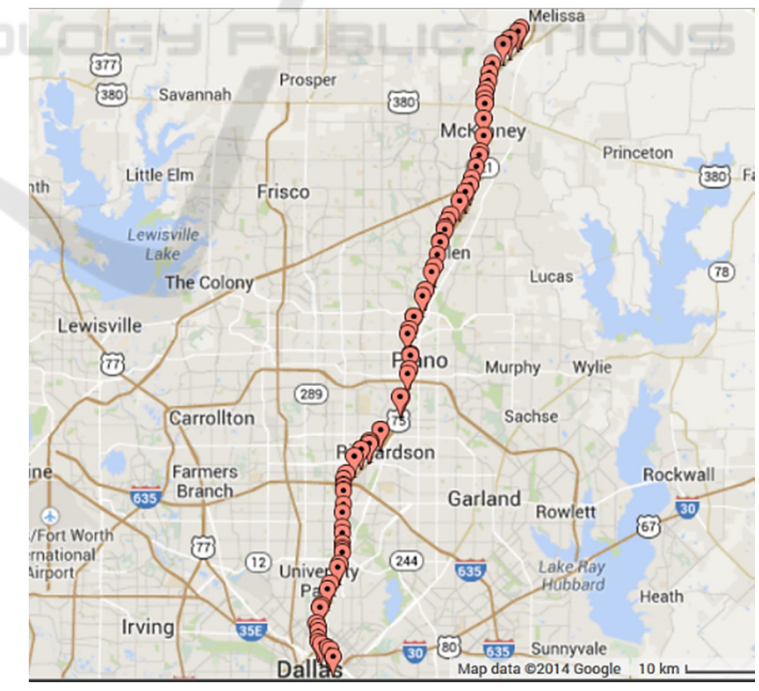

Figure 4: Layout of the Selected Freeway Stretch on US75. (Source: Google Maps). 


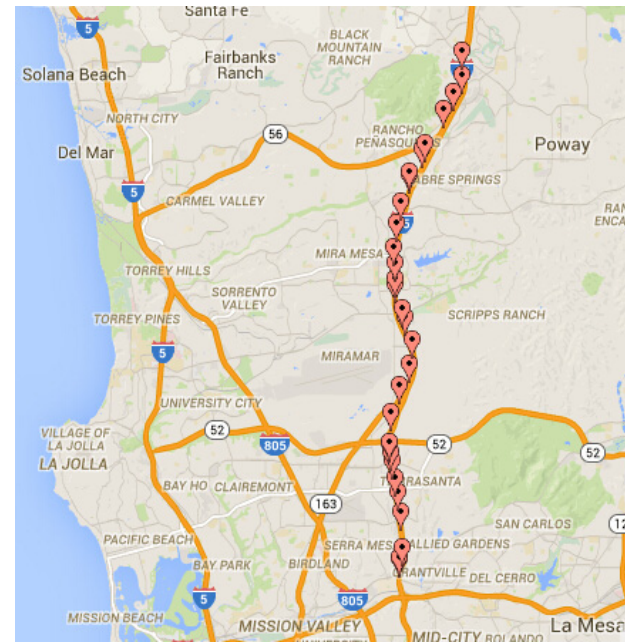

Figure 5: Layout of the Selected Freeway Stretch on I-15. (Source: Google Maps).

\subsection{Effect of Visibility and Weather Conditions}

This subsection describes the investigation of weather and visibility impacts on the cut-off speed (threshold) that is used to define the congested condition. The investigation was limited by the fact that data could not be divided into bins containing each weather condition and visibility level. Moreover, many bins had small amounts of data or no data at all. With this in mind, the mixture of linear regressions is proposed to pool data and estimate cut-off speeds, without sorting the data into clusters. In this subsection, we describe a speed model, featuring a mix of three linear regressions. Each linear equation describes a relationship between independent variables (visibility and weather) and the dependent variable, which is speed. In other words, instead of mixing three components with unchanged means, the speed model mixed three components whose means were a function of weather and visibility.

\subsection{Unified Model}

In order to get a unified model that is independent of the location or the speed limit, we did the following:

1. Weather conditions for the three data sets were consolidated based on precipitation. Weather conditions from all three data sets were then mapped into these weather groups. As shown in appendix A. Weather conditions from all three data sets were then mapped into these weather groups.
2. We put all the three datasets in one pool and did not include indicator variables that show the ID of the dataset.

3. The speed is normalized by dividing the speed at each road segment by the posted maximum speed at this segment.

The unified model has a response which is the normalized speed come from the three datasets and the predictors are the indicator variables for the weather groups and the visibility level.

In applying the mixture of three linear regression model, speed and visibility data were grouped by weather. Because the data set is huge and we cannot estimate the model parameters using the whole data set at once due to memory issues, a total of 7,000 random sample were then drawn randomly from each weather group, to construct a realization (dataset). Each random sample includes the speed and visibility level, together with indicator variables for the weather. Because speed distributions are skewed, the log-normal distribution is preferred to the normal distribution. Log speed was used as the response variable. Weather code and visibility were the explanatory variables (predictors). Coefficients of the predictors $\left(\beta_{1}, \beta_{2}, \beta_{3}\right)$, variance of each component $\left(\sigma_{1}^{2}, \sigma_{2}^{2}, \sigma_{3}^{2}\right)$ and proportions $\left(\lambda_{1}, \lambda_{2}, \lambda_{3}\right)$ of each component were estimated using the above iterative EM algorithm (Equations 3-6). This procedure was repeated 300 times by bootstrapping the sample construction without replacement. Final model parameters were the mean or median of all model coefficients. Once the final model was derived, we can observe the shift of the distribution mean with the weather condition and visibility level in the three regimes (free-flow, speed at capacity and congested). Given any combination of weather and visibility. The final model computes mean speeds for the three regimes. Furthermore, using the estimated model's parameters, the model computes Bayesian and 0.001 quantile cut-off speeds.

The estimated general model's parameters are shown in Table 2. As shown figure 6 the results are sensible because all weather groups have cut-off speeds lower or equal to the clear group. Moreover, the cut-off speed increases as visibility increases. We should mention that the cut-off speed for clear and light rain are very close so we can apply the cut-off speed of the clear condition at the light rain as well. Appendix B shows the speed matrix and the corresponding binary matrix after applying the proposed algorithm. 
Table 2: Unified model's parameters.

\begin{tabular}{|c|c|c|c|}
\hline & Congestion & Speed at capacity & Free flow \\
\hline 'Clear' (Intercept) & -0.9025 & -0.1947 & 0.0335 \\
'Visibility' & 0.0260 & 0.0229 & 0.0026 \\
'Medium Rain' & -0.0722 & -0.0024 & -0.0238 \\
'Heavy Rain' & -0.0398 & -0.0465 & -0.0308 \\
'freezing rain' & 0.2809 & -0.1134 & -0.0018 \\
'Snow' & 0.1754 & -0.0740 & -0.0149 \\
\hline$\sigma$ & 0.4881 & 0.1027 & 0.0680 \\
\hline$\lambda_{j}$ & 0.0846 & 0.1123 & 0.8028 \\
\hline
\end{tabular}

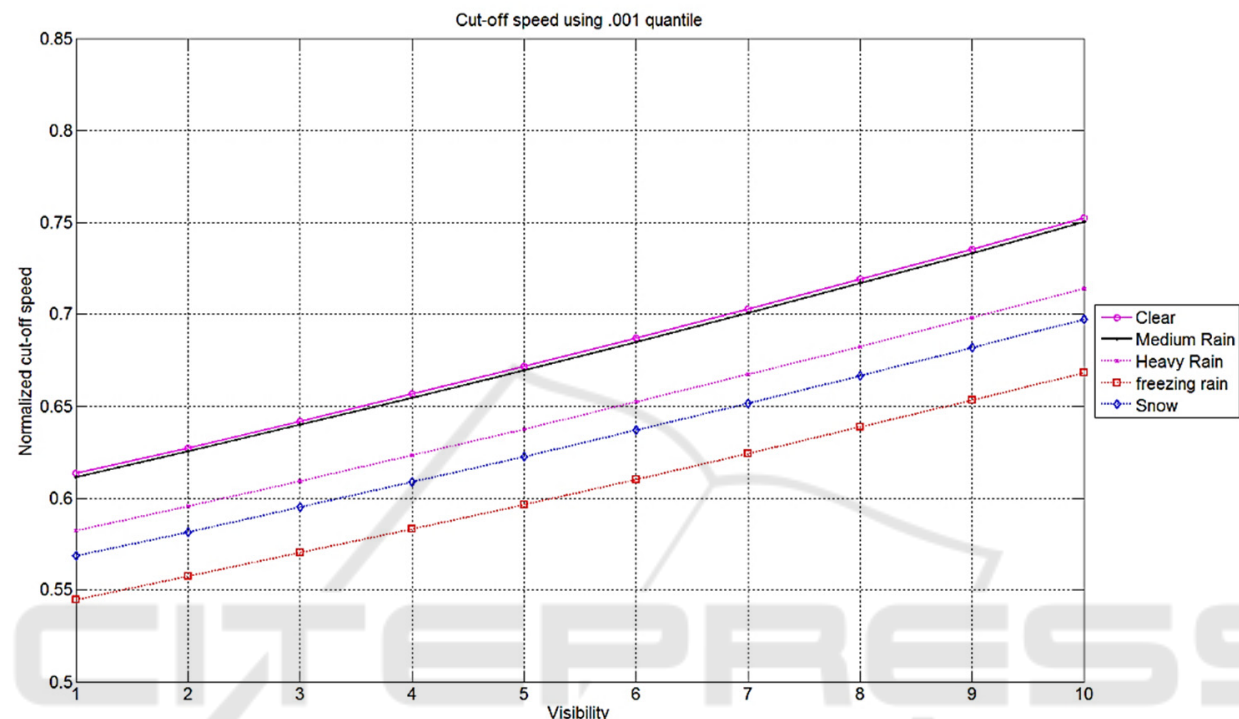

(a)

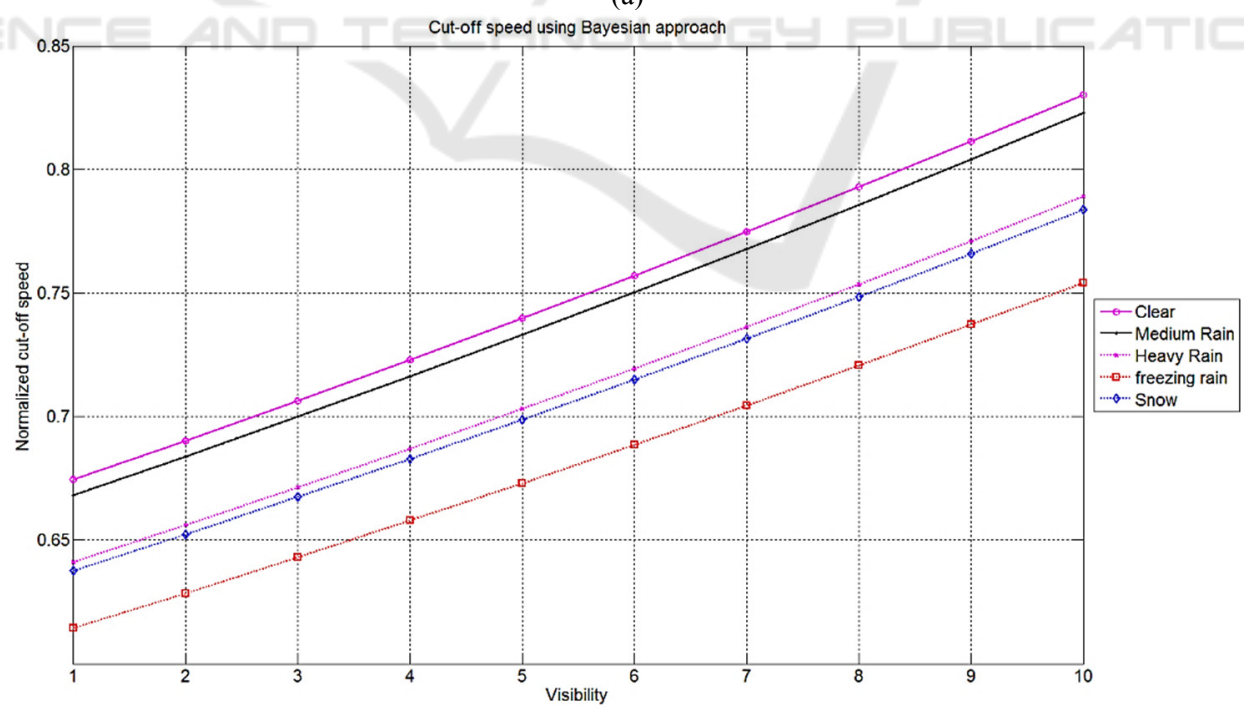

(b)

Figure 6: The Unified Model's Cut-off Speeds (a) Quantile, (b) Bayesian.

\subsection{Example Illustration}

Recall that, he model that explain the variation in normalized speed using the weather and visibility is shown in Equation (9) 


$$
\begin{aligned}
& \left(\log (\mathrm{y}) \mid \lambda_{1}, \lambda_{2}, \beta_{1}, \beta_{2}, \beta_{3}, \sigma_{1}, \sigma_{2}, \sigma_{3}\right)= \\
& \lambda_{1} \frac{1}{\sqrt{2 \pi} \sigma_{1}} \mathrm{e}^{\frac{\left(\log (y)-\mathrm{x}^{\mathrm{T}} \beta_{1}\right)^{2}}{2 \sigma_{1}{ }^{2}}}+\lambda_{2} \frac{1}{\sqrt{2 \pi} \sigma_{2}} \mathrm{e}^{\frac{\left(\log (\mathrm{y})-\mathrm{x}^{\mathrm{T}} \beta_{2}\right)^{2}}{2 \sigma_{2}{ }^{2}}}+ \\
& \frac{\llbracket\left(1-\lambda \rrbracket_{2}-\lambda_{1}\right) 1}{\sqrt{2 \pi} \sigma_{3}} \mathrm{e}^{\frac{\left(\log (\mathrm{y})-\mathrm{x}^{\mathrm{T}} \beta_{3}\right)^{2}}{2 \sigma_{3}{ }^{2}}},
\end{aligned}
$$

Where vector $x$ is the vector of weather conditions and visibility predictors, and $\mathrm{y}$ is the normalized speed. Here $\left(\mathrm{X}^{\mathrm{T}} \beta_{1}, \sigma_{1}\right),\left(\mathrm{X}^{\mathrm{T}} \beta_{2}, \sigma_{2}\right)$, and $\left(\mathrm{X}^{\mathrm{T}} \beta_{3}, \sigma_{3}\right)$ are the locations and spreads of the mixture components and $\left(\lambda_{1}, \lambda_{2}\right)$ are the mixture parameter.

The above table shows the equations that govern the locations of the three components are:

$$
\mu_{\text {Congestion }}=-0.9025+0.0260 * \text { Visibility }-
$$

$0.0722 *$ Medium Rain $-0.0398 *$ Heavy Rain +

$0.2809 *$ freezing rain $+0.1754 *$ Snow

$$
\mu_{\text {Speed at capacity }}=-0.1947+0.0229 *
$$

Visibility $-0.0024 *$ Medium Rain $-0.0465 *$ Heavy Rain $-0.1134 *$ freezing rain $0.0740 *$ Snow

$$
\mu_{\text {Free flow }}=0.0335+0.0026 * \text { Visibility }-
$$

$0.0238 *$ Medium Rain $-0.0308 *$ Heavy Rain -

$0.0018 *$ freezing rain $-0.0149 *$ Snow

Let's give an example to show how to come up with the Q quantile cut-off speed for given weather group and visibility level. . Based on the model the predictors' vector is as shown in Equation (13)

$X^{T}=$

[Visibility Medium Rain Heavy Rain freezing rain Snow] (13)

Assume the weather is "freezing rain" and the visibility is " 2 ", what is the Q quantile cut-off speed. Given the previous information, the predictors' vector is shown in equation (14),

$$
X^{T}=\left[\begin{array}{llllll}
1 & 2 & 0 & 0 & 1 & 0
\end{array}\right]
$$

Then the mean of speed at capacity component is calculated as shown in equation (15)

$\mu_{\text {Speed at capacity }}=-0.1947+0.0229 * 2-0.0024 *$
$0-0.0465 * 0-0.1134 * 1-0.0740 * 0$

Manipulating the above equation, we get -0.2623 as the mean of the speed at capacity component. Then using the Matlab command "norminv(Q, -0.2623, 0.1123 )" we get the Q quantile cut-off speed where 0.1123 is the standard deviation for the speed at capacity component. We should highlights that the standard deviation and the proportion parameters are constant and do not depend on the weather group or visibility.
Now, let assume we are interested in the .001 quantile at the "freezing rain" and the visibility is " 2 ". Using the Matlab command "norminv(.001, -0.2623 , 0.1123 )" we get the .001 quantile cut-off speed which is -0.6093 . -0.6093 is the cut-off speed on the $\log$ scale and the cut-off speed used to get the binary matrix is $\exp (-0.6093)=0.5437$.In the previous example, the .001 quantile cut-off speed is 0.5437 of the posted speed. In other words, the cut-off speed is $0.5437 * 65=35.3405 \mathrm{MPH}$ if the posted speed is 65 MPH.

\section{CONCLUSIONS}

This study developed models of speed distributions in free-flow, speed at capacity and congested traffic states using of mixture of linear regressions. To the best of our knowledge, this is the first methodology integrates the impact of weather and visibility into automated congestion identification. Moreover, this methodology is expected to be more portable because it is based on three different data set covers three various regions that hopefully represent the US. The proposed algorithm is expected to be the state of practice at many DOTs because of its simplicity, promising result and suitability to run in real-time scenarios. Because our algorithm precisely identifies the traffic congestion, both spatially and temporally, it is recommended as an important first step towards identifying and ranking bottlenecks.

\section{ACKNOWLEDGEMENTS}

This effort was funded by the Federal Highway Administration and the Mid-Atlantic University Transportation Center (MAUTC).

\section{REFERENCES}

Agarwal, M., Maze, T. H. \& Souleyrette, R. Impacts Of Weather On Urban Freeway Traffic Flow Characteristics And Facility Capacity. Proceedings of the 2005 Mid-Continent Transportation Research Symposium, 2005.

Arnott, R. \& Small, K. 1994. The Economics of Traffic Congestion. American Scientist, 82, 446-455.

Brilon, W. \& Ponzlet, M. 1996. Variability of Speed-Flow Relationships on German Autobahns. Transportation Research Record: Journal of The Transportation Research Board, 1555, 91-98. 
Chung, E., Ohtani, O., Warita, H., Kuwahara, M. \& Morita, H. Does Weather Affect Highway Capacity. In 5th International Symposium on Highway Capacity and Quality of Service, 2006 Yakoma, Japan.

Dailey, D. J. 2006. The Use of Weather Data to Predict Non-Recurring Traffic Congestion.

De Veaux, R. D. 1989. Mixtures of Linear Regressions. Computational Statistics \& Data Analysis, 8, 227-245.

Elhenawy, M., Chen, H. \& Rakha, H. A. Traffic Congestion Identification Considering Weather and Visibility Conditions using Mixture Linear Regression. Transportation Research Board 94th Annual Meeting, 2015.

Elhenawy, M. \& Rakha, H. 2013. Congestion Identification with Skewed Component Distributions Vtti.

Elhenawy, M., Rakha, H. A. \& Hao, C. An Automated Statistically-Principled Bottleneck Identification Algorithm (Asbia). Intelligent Transportation Systems - (Itsc), 2013 16th International Ieee Conference On, 69 Oct. 2013 2013. 1846-1851.

Faria, S. \& Soromenho, G. 2009. Fitting Mixtures Of Linear Regressions. Journal of Statistical Computation And Simulation, 80, 201-225.

Guiyan, J., Shifeng, N., Ande, C., Zhiqiang, M. \& Chunqin, Z. The Method Of Traffic Congestion Identification And Spatial And Temporal Dispersion Range Estimation. 2nd International Asia Conference On Informatics In Control, Automation And Robotics (Car), 2010 6-7 March 2010 2010. 36-39.

Ibrahim, A. T. \& Hall, F. L. 1994. Effect Of Adverse Weather Conditions On Speed-Flow-Occupancy Relationships.

Jianming, H., Qiang, M., Qi, W., Jiajie, Z. \& Yi, Z. 2012. Traffic Congestion Identification based on Image Processing. Intelligent Transport Systems, Iet, 6, 153-160.
Meead Saberi, K. \& Bertini, R. L. Empirical Analysis Of The Effects Of Rain On Measured Freeway Traffic Parameters.

Nookala, L. S. 2006. Weather Impact on Traffic Conditions And Travel Time Prediction. Doctoral Dissertation, University Of Minnesota Duluth.

Rakha, H., Farzaneh, M., Arafeh, M., Hranac, R., Sterzin, E. \& Krechmer, D. 2007. Empirical Studies On Traffic Flow In Inclement Weather.

Smith, B. L., Byrne, K. G., Copperman, R. B., Hennessy, S. M. \& Goodall, N. J. 2003. An Investigation Into The Impact Of Rainfall On Freeway Traffic Flow.

Sun, Z.-Q., Feng, J.-Q., Liu, W. \& Zhu, X.-M. Traffic Congestion Identification Based On Parallel Svm. Natural Computation (Icnc), 2012 Eighth International Conference On, 29-31 May 2012 2012. 286-289.

Sweet, M. 2011. Does Traffic Congestion Slow The Economy? Journal of Planning Literature, 26, 391-404.

Xu, L., Yue, Y. \& Li, Q. 2013. Identifying Urban Traffic Congestion Pattern from Historical Floating Car Data. Procedia - Social and Behavioral Sciences, 96, $2084-$ 2095.

\section{APPENDIX}

Table 3: Six weather groups.

\begin{tabular}{|c|r|}
\hline Groups & $\#$ \\
\hline Clear & 1 \\
\hline Light Rain & 2 \\
\hline Rain & 3 \\
\hline Heavy rain & 4 \\
\hline Freezing rain & 5 \\
\hline Snow & 6 \\
\hline
\end{tabular}

Figure 7 shows the speed matrix and the corresponding binary matrix after applying the proposed algorithm. The binary matrix will be further filtered to fill gaps and remove noise using image processing techniques. 

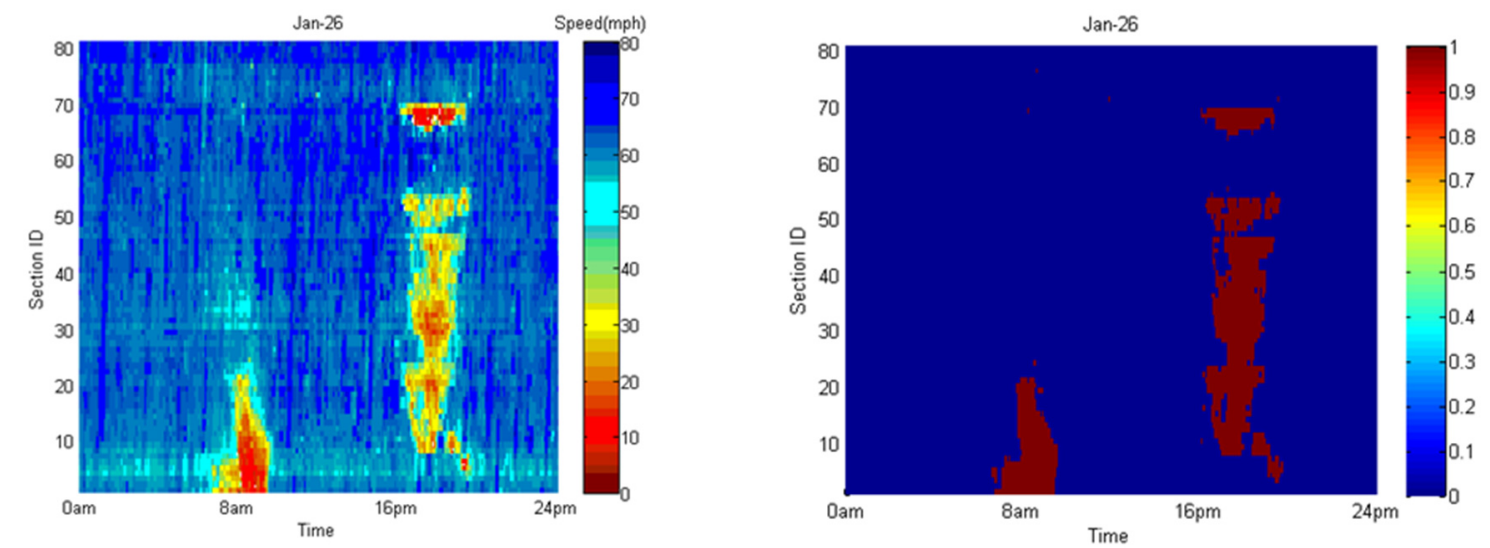

(a)
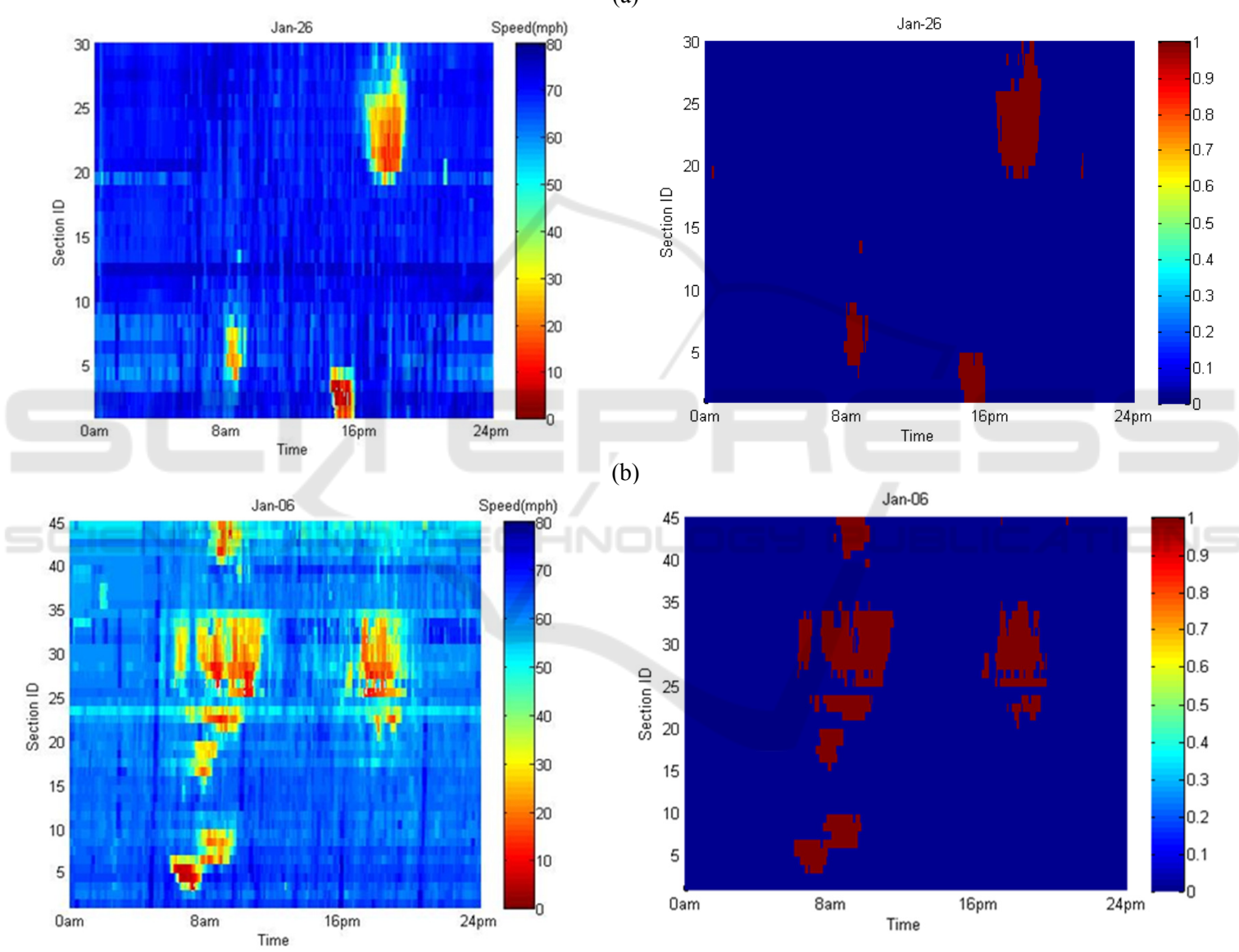

(b)

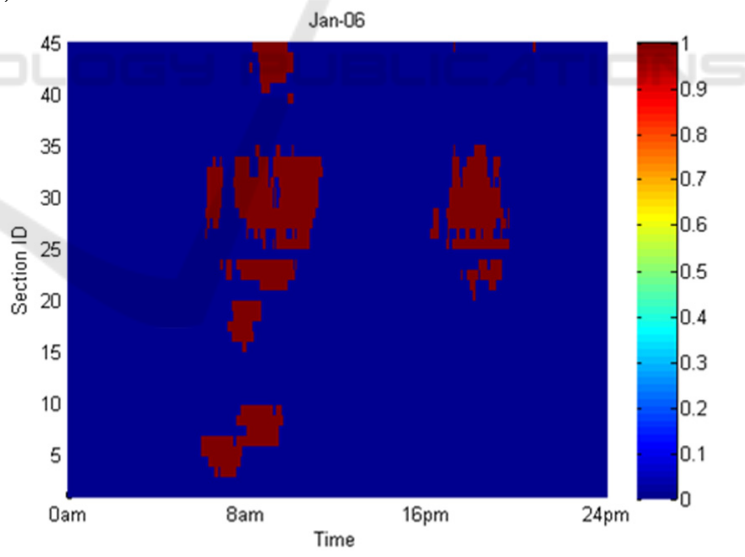

(c)

Figure 7: Speed (left) and Binary Matrix after Applying Algorithm (right); (a) TX; (b) CA; (c) VA. 